\title{
Study on pivot-point vibration of molecular bond-rupture events by quartz crystal microbalance for biomedical diagnostics
}

This article was published in the following Dove Press journal:

International Journal of Nanomedicine

23 January 2012

Number of times this article has been viewed

\author{
Yong J Yuan \\ Renjie Jia \\ Laboratory of Biosensing and \\ MicroMechatronics, School of \\ Materials Science and Engineering, \\ Southwest Jiaotong University, \\ Chengdu, Sichuan, People's \\ Republic of China
}

\begin{abstract}
Bond-rupture scanning for biomedical diagnostics is examined using quartz crystal microbalance (QCM) experiments and microparticle mechanics modeling calculations. Specific and nonspecific interactions between a microparticle and its binding QCM surface can be distinguished by gradually increasing the amplitude of driving voltage applied to QCM and monitoring its frequency changes. This research proposes a mechanical model of interactions between biological molecules and a QCM substrate surface. The mechanical force required to break a biotin-streptavidin bond was calculated through a one-pivot-point bottom-up vibration model. The bond-rupture force increases with an increase of the microparticle radius, the QCM resonant frequency, and the amplitude of driving voltage applied to the QCM. The significance of the research on biological molecular bond rupture is extremely important in characterizing microbial (such as cells and virus) specificity, due to the force magnitude needed to break bonds using a transducer.
\end{abstract}

Keywords: bond rupture, mechanical force, biomolecular binding energy spectra, quartz crystal microbalance (QCM)

\section{Introduction}

Molecular bond-rupture scanning is an effective method for the measurement of the energy level necessary to break a bond and distinguishing specific and nonspecific binding of biological molecules. Because of differences in energy levels, nonspecific binding can be eliminated by introducing energy mechanically through displacement of a resonant quartz crystal with quartz crystal microbalance (QCM). ${ }^{1}$ By increasing the voltage applied to QCM, the nonspecific binding breaks first with lower energy, followed by the specific binding. Due to the many advantages that a bond-rupture biosensor possesses, such as high efficiency, real-time, economy, and high sensitivity, it is applicable for a specific recognition immunoassay test, especially for the study of the interaction of biological molecules, detection of biological molecules, and real-time, online trace analysis of biological substances.

Based on the intention to explore biomolecular binding, there have been a number of reported successful bond-rupture tests with varied results. Yuan et $\mathrm{al}^{1}$ applied both static scanning and dynamic scanning methods to monitor the interaction of streptavidin-coated polystyrene microspheres and a biotinylated QCM surface. They simultaneously measured the frequency change and noise signals from the rupture of biotin-streptavidin bonds. Dultsev et $\mathrm{al}^{2}$ and Cooper et $\mathrm{al}^{3}$ reported successful bondrupture tests and successful noise capture with microspheres. The specific binding that Dultsev et $\mathrm{al}^{2}$ used was also biotin-streptavidin, whereas Cooper et $\mathrm{al}^{3}$ used a herpes 
simplex virus-glycoprotein D binding system. Edvardsson et $\mathrm{al}^{4}$ attempted bond rupture using a modified QCM-D instrument but failed to rupture the bonds.

To obtain the relationship between driving voltage applied to a resonator and the mechanical force of bond rupture, this paper presents a mechanical model - one-pivot-point bottom-up vibration - of the principle of molecular bond rupture, theoretical analysis, and solution of the mechanical force generated by QCM. Based on the results obtained, comprehensive conclusions are discussed. More importantly, a pivot vibration model will be used not only to evaluate the bond-rupture force but also to characterize the surface bonding mechanism of biotin and streptavidin. The movement of a microsphere on a QCM surface can be described as translation, ${ }^{5}$ rotation, ${ }^{6}$ and pivot vibration. ${ }^{7}$ Based on the classification of pivot structures, there are three major types in pivot vibration models, such as one point, ${ }^{8}$ two points, ${ }^{9}$ and a bulk. ${ }^{7}$ Figure 1 shows the relationship of all vibration modes and a brief summary of concepts in the pivot vibration mode.

One-pivot-point vibration was discussed $^{8}$ in the study of frequency of a beetle-style scanning probe microscope's scanhead. Voltages applied to electrodes induced transverse bending and axial compression or elongation via the inverse piezoelectric effect, controlling the motion of the scanning probe microscopy (SPM) scanhead. Brukman and Carpick ${ }^{8}$ identified a new vibration mode of the head: pivot vibration, shown in Figure 1A. In addition, they proposed the pivot vibration frequency with the equation as follows:

$$
f_{\text {pivot }}=\sqrt{\frac{E I \pi^{2}}{64 L^{3}}} \div \sqrt{\frac{J+M_{\text {disk }} R^{2}}{R^{2}}+M_{\text {leg }}}
$$

where $R$ is the radial distance from the legs to the center of the disk, $M$ is the true mass of the disk and probe equipment,
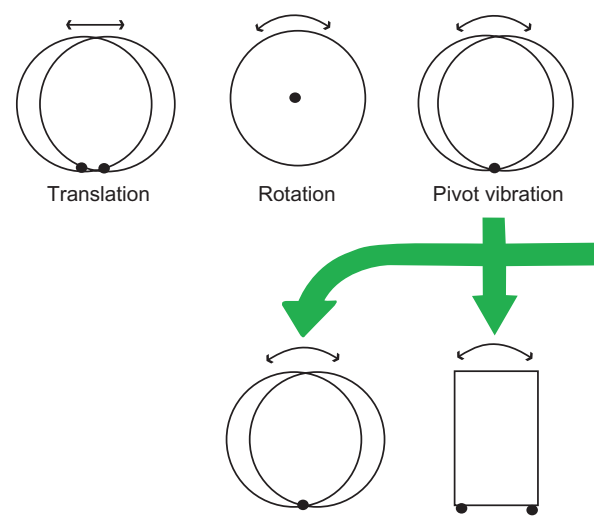

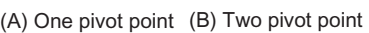

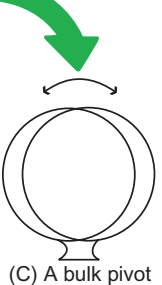

Figure I The schematic diagram of vibration modes. and $J$ is the mass polar moment of inertia of the disk and probe equipment about the disk's central axis. $M_{\text {leg }}=A_{x s} L \rho$, where $A_{x s}$ is the cross-sectional area of a leg.

Two-pivot-point vibration was discussed in the n-stories building model in an earthquake. The common analytical method is seismic isolation, ${ }^{9}$ as shown in Figure 1B. The base in the isolation system was simplified into a two-point pivot. The equation of motion for the structure is expressed in the matrix form as:

$\left[M_{s}\right]\left\{\ddot{x}_{s}\right\}+\left[C_{s}\right]\left\{\dot{x}_{s}\right\}+\left[K_{s}\right]\left\{x_{s}\right\}=-\left[M_{s}\right]\{r\}\left\{\ddot{x}_{b}+\ddot{x}_{g}\right\}$

where $\left[M_{s}\right],\left[C_{s}\right]$, and $\left[K_{s}\right]$ are the mass, damping, and stiffness matrices of the structure, respectively; $\left\{x_{s}\right\}-\left\{x_{1}, x_{2}, \ldots x_{N}\right\}^{\mathrm{T}}$, $\left\{\dot{x}_{s}\right\}$, and $\left\{\ddot{x}_{s}\right\}$ are the unknown relative floor displacement, velocity, and acceleration vectors, respectively; $\ddot{x}_{b}$ and $\ddot{x}_{g}$ are the relative acceleration of base mass and earthquake ground acceleration, respectively; and $\{r\}$ is the vector of influence coefficients.

Murthy Peri and Cetinkaya ${ }^{7}$ did an experiment on noncontact adhesion measurement and compared the rolling motion with the axial displacement of the bond. A chemical bond was treated as a bulk pivot, which is shown in Figure 1C. They calculated the natural frequency of this oscillator, which is shown in the following equation:

$$
\omega_{n}=\frac{1}{r^{3 / 2}} \sqrt{\frac{45}{4} \frac{W_{A}}{\rho}}
$$

where $W_{A}$ is the work of adhesion, $\rho$ is the mass density of the particle material, and $r$ is the particle radius. Pivot vibration is the combination of vibration and rotation. The number of pivot points should be chosen based on experimental conditions.

\section{Methodology and simulation mode A piezoelectric model of thickness shear mode}

One-pivot-point bottom-up vibration was proposed to analyze the interaction between a microsphere and a substrate in bond-rupture experiments. QCM converts the mass changes of the quartz crystal electrode surface into an output signal's frequency changes ${ }^{10}$ by a crystal oscillator circuit ${ }^{11}$ and then produces highly precise data from a computer and other auxiliary equipment. The commonly used AT-cut quartz crystal oscillates almost in a thickness shear mode (TSM). ${ }^{12}$ When the crystal vibrates, one of the two diagonals on the side of the crystal stretches while the other shortens, and the 
wave nodal plane goes through the central chip, paralleling with the main plane. This shear vibration mode, which is relevant to the thickness, is shown in Figure 2A. The oscillating frequency of this mode is very high, and the chip can be ground to be very thin.

In order for a mechanical force to break a bond, surface displacement of the AT-cut TSM quartz crystal was first taken into account. Most AT-cut TSM piezoelectric quartz crystals are excited with a vertical field. Under electric field $E$ and along the $y$ direction, thickness shear vibration was generated on the $z$ surface, where the piezoelectric constant is $e_{26}$ and elastic stiffness constant is $c_{66}^{D}$. The density of the quartz is $\rho$. The displacement of the quartz along the $x$ axis, $u$, is described by the one-dimensional wave equation:

$$
\frac{\partial^{2} u}{\partial t^{2}}=c^{2} \frac{\partial^{2} u}{\partial y^{2}}
$$

Here, $c$ is the wave velocity $\left(c=\sqrt{c_{66}^{D} / \rho}\right)$. The general solution for the wave equation is:

$$
u=(A \cos k y+B \sin k y) \exp (j \omega t)
$$

Here, $k=\omega / c$. The fourth-class piezoelectric equation of the AT-cut TSM quartz is defined as follows: ${ }^{13} E_{2}=-h_{26} S_{6}+D_{2} / \varepsilon_{22}$ and $T_{6}=c_{66}^{D} S_{6}-h_{26} D_{2}$. Here, $E_{2}$ is the electric field along the $y$ direction, and $T_{6}$ is the shear stress in the $x y$ plane. $h_{26}$ is the piezoelectric constant matrix, and $\varepsilon_{22}$ is the dielectric constant matrix. Shear strain in the $x y$ plane is $S_{6}=\partial u / \partial y$, and $D_{2}$ is the
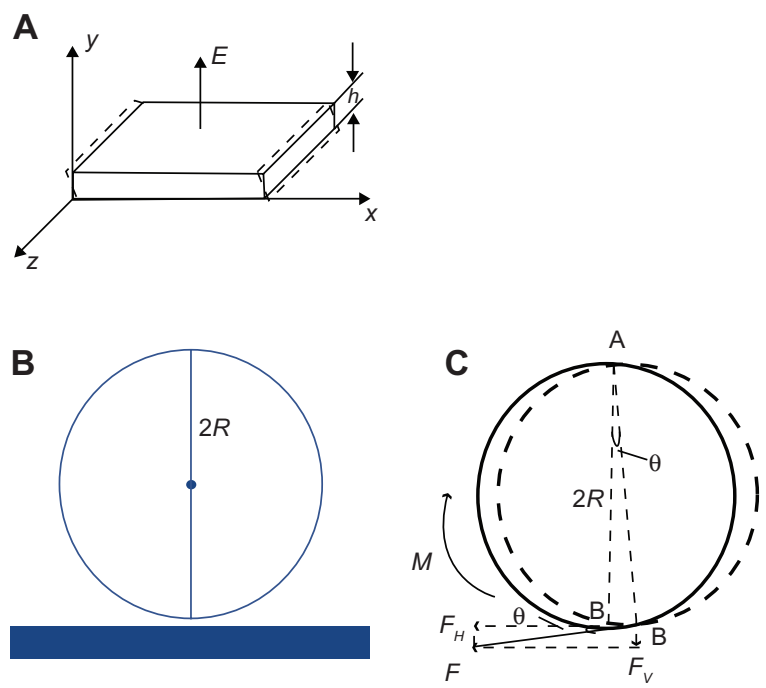

Figure 2 The mechanical model of the interaction of the sphere and with quartz crystal microbalance platform. (A) The thickness shear oscillation of a piezoelectric quartz crystal. $E$ is the electric field direction of the piezoelectric effect. $h$ is the thickness of the quartz. (B) Diagram of the flexible connection. (C) The mechanical model of the sphere rotating around the vertex. electric displacement along the y direction. If the electric field $E_{2}$ is given as $E_{2}=E=E_{0} \exp (j \omega t)$, then the electric displacement $D_{2}$ is:

$$
\begin{aligned}
D_{2}= & \varepsilon_{22}\left(E_{2}+h_{26} \mathrm{~S}_{6}\right)=\varepsilon_{22}\left(E_{0}+h_{26}(-A k \sin k y+B k \cos k y)\right) \\
& \times \exp (j \omega t)=D_{0} \exp (j \omega t)
\end{aligned}
$$

and the shear stress

$$
T_{6}=\left(c_{66}^{D}(-A k \sin k y+B k \cos k y)-h_{26} D_{0}\right) \exp (j \omega t) .
$$

At the free surface, the boundary condition is $T_{6 \cdot y=0}=T_{6 \cdot y=1}=0 . A$ and $B$ in Equation (5) can be substituted. The solution for the AT-cut TSM crystal wave equation is:

$$
u=\frac{h_{26} D_{0}}{c_{66}^{D} k}\left[\frac{\cos k(h-y)-\cos k y}{\sin k h}\right] \exp (j \omega t)
$$

The $x$-axial displacement $u$ of the chip surface when $y=h$ is $u=A \exp (j \omega t)) . A=h_{26} D_{0} / c_{66}^{D} k[1-\cos k h / \sin k h]$ is the maximum displacement of a TSM chip.

\section{A mechanical model of the interaction of microspheres and QCM surface}

If a streptavidin-coated microsphere was bound on the QCM surface by biotin-streptavidin interaction, ${ }^{1,14}$ a uniform microsphere was approximately attached to the QCM surface through a flexible bond, as shown in Figure 2B. The sphere's vibration can be considered as a mechanical mode, as shown in Figure 2C. The surface displacement of QCM is a few nanometers or several tens of nanometers, as reported by Edvardsson et al. ${ }^{15}$ With regard to the microsphere radius at a micron level, it can be assumed that the bottom of the microsphere vibrated with the quartz crystal. In order to take pivot vibration into account, it is necessary to postulate the fixed top point of a microsphere. The microsphere vibrated around the vertex A, as shown in Figure $2 \mathrm{C}$. The displacement of the bottom $\mathrm{B}$ is determined by the horizontal displacement of the piezoelectric plate's surface, as discussed previously as $u$. Then, the line speed of point $\mathrm{B}$ is given as follows: $v(t)=d u(t) / d t=j A \omega \exp (j \omega t)$. The angular acceleration of point $\mathrm{B}$ is $\beta(t)=1 / R d v(t) / d t=-A / R \omega^{2} \exp (j \omega t)$. The rotational moment around point $A$ is $M(t)=I \beta(t)=-I A / R \omega^{2} \exp (j \omega t)$. Here, $I$ is the moment of inertia rotating around point $\mathrm{A}$. The calculation of the moment of inertia rotating around vertex $\mathrm{A}$ is given as follows: $I=\int_{0}^{2 R} l^{2} d m=6 / 5 m R^{2}$. Then, the rotational torque is $M(t)=-I A / R \omega^{2} \exp (j \omega t)=-6 / 5 m R A \omega^{2} \exp (j \omega t)$. Moment $M=F l=F \cdot 2 R$, so the force $F$ that the TSM chip 
drives the microsphere to vibrate at (as is shown in Figure $2 \mathrm{C}$ ) is $F(t)=M(t) / 2 R=-3 / 5 m A \omega^{2} \exp (j \omega t)$. The angle between $F$ and horizontal direction is $\tan \theta=\mu / 2 R=A / 2 R \exp (j \omega t)$ and $\sin \theta=u / \sqrt{u^{2}+4 R^{2}}=A \exp (j \omega t) / \sqrt{A^{2} \exp (j \omega 2 t)+4 R^{2}}$. Therefore, the vertical component of $F$ is:

$$
F_{v}(t)=F(t) \sin \theta=-\frac{3}{5} m A^{2} \omega^{2} \frac{\exp (j 2 \omega t)}{\sqrt{A^{2} \exp (j 2 \omega t)+4 R^{2}}}
$$

when $\exp (j 2 \omega t)=1$, the vertical component $F_{v}$ reaches the maximum (ie, $t=n \pi / \omega, \mathrm{n}=0,1,2,3 \ldots \ldots$ ):

$$
\left|F_{v \max }\right|=\frac{3}{5 \sqrt{A^{2}+4 R^{2}}} m A^{2} \omega^{2}
$$

As is shown in Figure 3, bond-rupture events happened between the microspheres and QCM. The forces exerted on the molecular bond include $F^{\prime}$ by the microsphere, the horizontal force $F_{q h}$, and the vertical force $F_{q v}$ by QCM surface and gravity $G_{2}$ (negligible). Given that the molecular bond was approximately rigid, the maximum vertical force $F_{B R}$ exerted on the molecular bond by the microsphere will be equal to the vertical component of $F$ in value.

$$
\left|F_{B R \max }\right|=\left|-F_{v \max }\right|=\frac{3}{5 \sqrt{A^{2}+4 R^{2}}} m A^{2} \omega^{2}
$$

Equation (9) is a generally applicable formula and is suitable for most macromolecular models, such as biotin-coated polystyrene with streptavidin and streptavidin-coated polystyrene with biotin. For further discussion, select streptavidin-coated polystyrene microsphere binding biotin as the interaction system. The microsphere's mass is $m=4 / 3 \pi \rho R^{3}$. Here, polystyrene's density $\rho$ is $1050 \mathrm{~kg} / \mathrm{m}^{3} .{ }^{16}$ Borovsky et $\mathrm{al}^{17}$ measured the relationship between the amplitude of the QCM and the voltage by experiments: $A=C Q V_{d}$, where $C=1.4 \times 10^{-12}$ is a constant determined experimentally, $Q$ the QCM merit factor ( $Q$ factor), and $V_{d}$ the driving voltage.

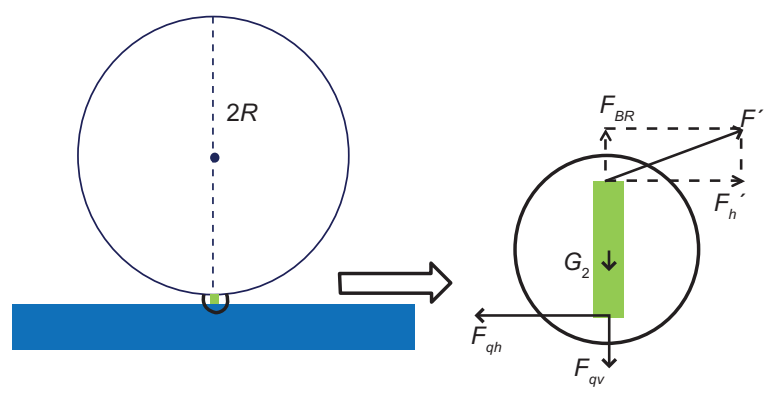

Figure 3 Stress analysis of a molecular bond.
Therefore, we can find the relationship between bond-rupture force and particle radius, the applied voltage, and frequency at the QCM electrode center.

$$
F_{B R}=\frac{4 \pi \rho R^{3}\left(C Q V_{d}\right)^{2} \omega^{2}}{5 \sqrt{\left(C Q V_{d}\right)^{2}+4 R^{2}}}=\frac{16 \pi^{3} \rho R^{3}\left(C Q V_{d}\right)^{2} f_{0}^{2}}{5 \sqrt{\left(C Q V_{d}\right)^{2}+4 R^{2}}}
$$

It is worth mentioning that the mass of streptavidin-coated polystyrene microsphere contains two parts: the mass of polystyrene microsphere $m_{P S}$ and surface-coating streptavidin $n_{S A} \cdot m_{S A}$. But the mass of surface-coating streptavidin is too small to be considered compared with polystyrene microsphere. So the bond rupture force we get in Equation (10) will be almost the same, without any significant difference.

\section{Results and discussion}

\section{Effect on bond-rupture force $F_{B R}$}

If QCM resonance frequency $\left(f_{0}\right)$ was $10 \mathrm{MHz}, Q$ factor $(Q)$ 2500 , and drive voltage $\left(V_{d}\right) 10 \mathrm{~V}$, the amplitude $(A)$ would be $35 \mathrm{~nm}$, according to $A=C Q V_{d}$. Under this scenario, the bond-rupture force $F_{B R}$ (Equation (10)) can be simplified as $F_{B R}=1.274 \times 10^{4} R^{3} / \sqrt{1.225 \times 10^{-15}+4 R^{2}}$. The supporting document of Appendix 1 shows the relationship between $F_{B R}$ and the radius $R$ at the QCM electrode center.

If the driving voltage and the resonant frequency are constants, the bond-rupture force provided by QCM will increase with the microsphere radius. The force to break a single biotin-streptavidin molecular bond, as measured by Grubmuller et al, ${ }^{18}$ was $0.3 \mathrm{nN}$. If the microsphere radius is less than a critical value $R$, the mechanical force that QCM provides will not break the bond. If the QCM resonance frequency is $10 \mathrm{MHz}, Q$ factor 2500 , and driving voltage $10 \mathrm{~V}$, then the critical value is $0.217 \mu \mathrm{m}$. So the mechanical force by QCM vibration will be not enough to break such a bond, due to there being a microsphere of less than $0.217 \mu \mathrm{m}$ radius. The bondrupture experiment will be successful only if the microsphere radius is larger than the critical value. This is a reason why upon increasing the driving amplitude after adsorption of a significant amount of $200 \mathrm{~nm}$ streptavidin-coated polystyrene spheres no desorption was observed. ${ }^{4}$ Comprehensive analysis of bond-rupture events should be carried out to determine the essential critical values and experimental parameters needed. Therefore, an appropriate microsphere radius is one of the most important selection criteria in bond-rupture experiments. ${ }^{14}$ The others, such as QCM resonant frequency and driving voltage, will be discussed here.

If the microsphere radius $(R), \mathrm{QCM} Q$ factor $(Q)$, and driving voltage $\left(V_{d}\right)$ are chosen as $1 \mu \mathrm{m}, 2500 \mathrm{~V}$, and $10 \mathrm{~V}$, 
respectively, the bond rupture force $F_{B R}$ (Equation (10)) can be simplified as $F_{B R}=6.370 \times 10^{-23} f_{0}^{2}$. The supporting document of Appendix 2 shows the relationship between $F_{B R}$ at the QCM electrode center and its resonant frequency $f_{0}$. In this situation, the bond-rupture force provided by QCM increases with the resonant frequency dramatically, due to being proportional to $f_{0}^{2}$. If the resonant frequency is less than a critical value $f_{0}$, the mechanical force that QCM provides will not break the bond.

When the particle radius is fixed to $1 \mu \mathrm{m}$, the $Q$ factor 2500 , and the driving voltage $10 \mathrm{~V}$, then the critical value is $2.17 \mathrm{MHz}$. That is, when the resonance frequency is less than $2.17 \mathrm{MHz}$, the mechanical force that QCM provides will not break the biotin-streptavidin molecular bond. So under the conditions that the voltage amplitude and particle radius are fixed, only when the resonance frequency is larger than a certain value will the bond-rupture experiment succeed. Therefore, an appropriate resonance frequency should be selected in bond-rupture experiments.

If the microsphere radius $(R)$, QCM $Q$ factor $(Q)$, and resonant frequency $\left(f_{0}\right)$ were chosen as $1 \mu \mathrm{m}$, $2500 \mathrm{MHz}$, and $10 \mathrm{MHz}$, respectively, then the bondrupture force $F_{B R}$ (Equation (10)) can be simplified as $F_{B R}=1.274 \times 10^{-10} V_{d}^{2} / \sqrt{1.225 \times 10^{-5} V_{d}^{2}+4}$. The supporting document of Appendix 3 shows the relationship between $F_{B R}$ at the QCM electrode center and its driving voltage $V_{d}$ Under this circumstance, the bond-rupture force provided by QCM increases with driving voltage. If driving voltage is less than a critical value $V_{d}$, the mechanical force due to QCM vibration will not break the bond.

If the microsphere radius, $Q$ factor, and resonant frequency are constants, such as $1 \mu \mathrm{m}, 2500 \mathrm{MHz}$, and $10 \mathrm{MHz}$, respectively, then the critical value of QCM driving voltage is $2.17 \mathrm{~V}$. The vibrating QCM should be driven at least $2.17 \mathrm{~V}$, and then a streptavidin-coated microsphere can fly off the QCM surface. Therefore, an appropriate QCM driving voltage is one of the most important selection criteria in bond-rupture experiments.

\section{Minimum bond-rupture force}

Comparison of QCM resonant frequency, driving voltage, and microsphere radius used for bond-rupture experiments by three research groups is summarized in Table 1 . The $Q$ factors of QCM were, respectively, 2500, 4000, and 5000, according to Edvardsson et al, ${ }^{4}$ Yuan et al, ${ }^{1}$ and Dultsev et al. ${ }^{2}$ According to Equation (10), the magnitude of the bondrupture force of the respective groups was also calculated, as shown in Table 1.
Table I Comparison of bond-rupture force $F_{B R}$ with regard to quartz crystal microbalance resonant frequency $f_{0}$, applied potential $V_{d}$, and microsphere radius $R$

\begin{tabular}{llll}
\hline & $\begin{array}{l}\text { Edvardsson et al's } \\
\text { research group }\end{array}$ & $\begin{array}{l}\text { Yuan et al's' }^{\prime} \\
\text { research group }\end{array}$ & $\begin{array}{l}\text { Dultsev et al's } \\
\text { research group }\end{array}$ \\
\hline$f_{0} / \mathrm{MHz}$ & 5 & 10 & 15 \\
$V_{d} / \mathrm{N}$ & 10 & 3.5 & 6 \\
$R / \mu \mathrm{m}$ & 0.1 & 3 & 2.5 \\
$F_{B R} / \mathrm{nN}$ & 0.016 & 17.983 & 129.016 \\
\hline
\end{tabular}

The same biotin-streptavidin interaction was carried out by three groups, but the calculated bond-rupture force of the first group was less than $0.3 \mathrm{nN}$, a minimum threshold of breaking a biotin-streptavidin molecular bond by QCM. This explains why Edvardsson et al failed to break the specific binding. The other two groups' bond-rupture forces were much greater than $0.3 \mathrm{nN}$. Here, the proposed one-pivot-point bottom-up vibration model is successfully demonstrated for bond-rupture events.

Figure 4 illustrates a schematic interaction between streptavidin coated on a polystyrene microsphere and biotin immobilized on a QCM surface, according to a resonance frequency profile of bond-rupture scanning measured by Yuan et al. ${ }^{1}$ The characteristics of QCM were $Q$ factor of 4000 and resonant frequency of $10 \mathrm{MHz}$, and microspheres of $3 \mu \mathrm{m}$ radius were tested.

As shown in Figure 4, driving voltage increased from $3.5 \mathrm{~V}$ to $12 \mathrm{~V}$. Specific binding broke gradually, which was caused by energy levels of specific bindings. If different energy levels were attributed to biotin being immobilized on a QCM gold surface and interacting with one streptavidin biomolecule on a polystyrene microsphere's surface, the one-pivot-point bottom-up vibration model can be further developed to determine how a microsphere is attached to a

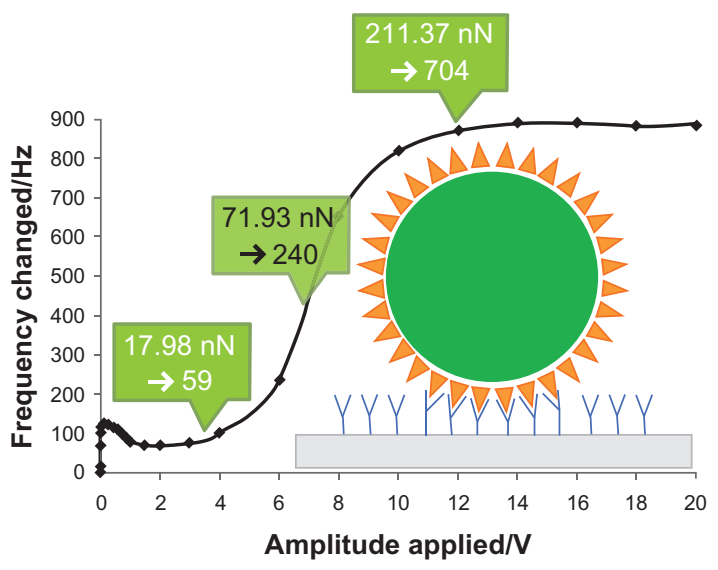

Figure $4 \mathrm{~A}$ model of interaction between streptavidin and biotin. 
QCM surface through multiple streptavidin-biotin bonds. The microsphere will not disengage from the QCM surface until all biotin-streptavidin bonds rupture.

According to Equation (10), the bond-rupture force of our group experiment ${ }^{1}$ was $17.98 \mathrm{nN}$ at $3.5 \mathrm{~V}$. Single biotin-streptavidin molecular bond strength was reported as $0.3 \mathrm{nN},{ }^{18}$ so the number of streptavidin-biotin bonds ruptured is approximately $17.98 / 0.3=59$ at $3.5 \mathrm{~V}$. As indicated in Figure 4, the calculated number of streptavidin-biotin bonds ruptured is, respectively, 240 and 704 at $7 \mathrm{~V}$ and $12 \mathrm{~V}$, due to the respective bond rupture of energy levels of $71.93 \mathrm{nN}$ and $211.37 \mathrm{nN}$. It showed a wide range of possible numbers of streptavidin-biotin bonds from 59 to 704 . There was a dramatic increase of resonant frequency at $7 \mathrm{~V}$, which is evidence that a majority of streptavidin-biotin bonds were ruptured by the QCM bond rupture of mechanical energy level of $71.93 \mathrm{nN}$, corresponding to 240 streptavidin-biotin bonds.

\section{Biotin-streptavidin binding mechanism}

A streptavidin molecule has four biotin binding sites, and the number of streptavidin molecules involved in binding is $n_{s}=n_{b} / \bar{n}$. Here, $n_{b}$ is the total number of bindings (ie, the number of biotin molecules) and $\bar{n}$ is the average number of binding sites of streptavidin $(0<\bar{n} \leq 4)$. When comparing the size of a streptavidin molecule with a polystyrene microsphere, streptavidin is supposed to be evenly distributed on the polystyrene surface. As shown in Figure 5A, the contact area $S$ between the microsphere and substrate can be alternated by $n_{s} a b, n_{s} a c$, or $n_{s} b c$, where $[a b c]$ are dimensions of a streptavidin molecule [a b c] $=\left[\begin{array}{lll}5.62 & 8.50 & 5.17\end{array}\right] \mathrm{nm} .{ }^{19}$

So the minimum contact area is $S_{\min }=n_{b} a c / \bar{n}$ and the maximum contact area is $S_{\max }=n_{b} a b / \bar{n}$. According to the spherical crown area formula $S=2 \pi R H, H=S / 2 \pi R, R$ is

A

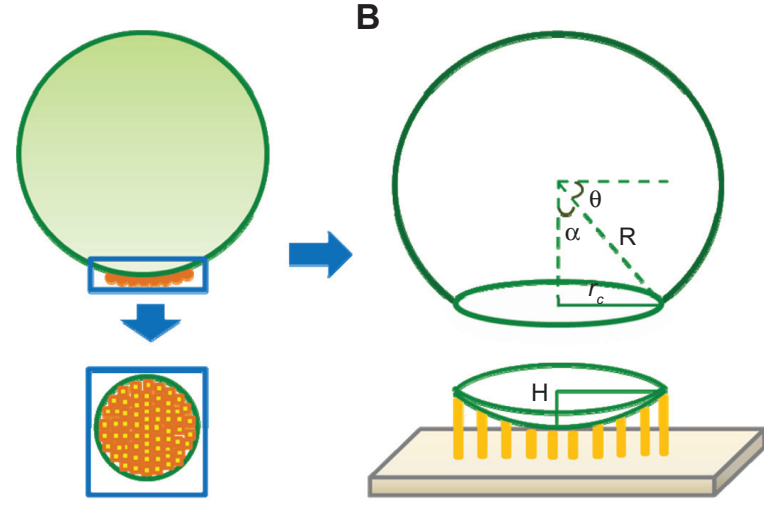

Figure $\mathbf{5}$ The binding sites calculation model. (A) Streptavidin molecules on the polystyrene microsphere surface. (B) The schematic diagram of the microsphere's spherical crown.
Table 2 Calculation of number of streptavidin binding sites at different voltages

\begin{tabular}{llllll}
\hline Contact model & $\boldsymbol{V}_{\mathbf{d}} / \mathbf{V}$ & $\mathbf{n}_{\mathrm{b}}$ & $\mathbf{S} / \mathbf{n m}^{2}$ & $\boldsymbol{H} / \mathbf{n m}$ & $\overline{\boldsymbol{n}}$ \\
\hline Minimum & 3.5 & 59 & 1714 & 0.09 & 0.2 \\
& 7 & 240 & 6973 & 0.37 & 0.9 \\
\multirow{3}{*}{ Median } & 12 & 704 & 20455 & 1.09 & 2.7 \\
& 3.5 & 59 & 2593 & 0.14 & 0.4 \\
& 7 & 240 & 10547 & 0.56 & 1.4 \\
Maximum & 12 & 704 & 30937 & 1.64 & 4.1 \\
& 3.5 & 59 & 2818 & 0.15 & 0.4 \\
& 7 & 240 & 11465 & 0.61 & 1.5 \\
& 12 & 704 & 33630 & 1.79 & 4.5 \\
\hline
\end{tabular}

the microsphere radius, $H$ is the height of a spherical crown, which must meet $H=l$, as illustrated in Figure 5B, and $l$ is the biotin-streptavidin bond length $0.4 \mathrm{~nm} .{ }^{18}$ Therefore, the average number of streptavidin binding sites must satisfy:

$$
\bar{n}=\frac{n_{b} a b}{2 \pi R l}
$$

According to our previous result ${ }^{1}$ of static-mode bondrupture of biotin and streptavidin, the average streptavidin binding sites can be predicted by a number of biotin-streptavidin bonds at three different voltages, as shown in Figure 4. This leads to streptavidin being immobilized by three types of orientation, resulting in minimum, median, and maximum contact areas. The detailed results are listed in Table 2.

The average binding sites of the specific binding that broke at $3.5 \mathrm{~V}$ should satisfy the inequality $0.2<\bar{n}<0.4$, which meets the condition $0<\bar{n} \leq 4$. At $7 \mathrm{~V}$ of the turning point of the mass changed profile, as shown in Figure 4, the majority of specific bindings (biotin-streptavidin) were ruptured at this point. It indicates an average binding site of $0.9 \sim 1.5$. The data at $12 \mathrm{~V}$ showed an average binding site number at least larger than 2.7, and even larger than 4 . This is clearly evidence that the interaction of biotin and streptavidin is supposed to be a one-binding-site model.

\section{Conclusion}

Molecular bond-rupture scanning is an advanced highsensitivity immunoassay biosensing technology, the development of which provides a new and efficient detection method for biomedical diagnostics and environmental monitoring. The analysis of mechanical force is instructive. In this research, emphasis is placed on the theoretical analysis of the relationship between the mechanical force provided by QCM and the several influencing factors at a nanoscale level. The selection of microparticle radius, QCM resonance frequency, and voltage 
amplitude in the biosensor should be considered carefully. The results have given great significance to theoretical research and practical applications of a bond-rupture biosensor, paving the way for extensively spreading to related areas.

\section{Acknowledgments}

This work was funded by the National Natural Science Foundation of China to YJY under General Program Fund No 30870664. Thanks are given to all members of the Southwest Jiaotong University Laboratory of Biosensing and MicroMechatronics for invaluable discussions.

\section{Disclosure}

The authors report no conflicts of interest in this work.

\section{References}

1. Yuan YJ, van der Werff MJ, Chen H, Hirst ER, Xu WL, Bronlund JE. Bond rupture of biomolecular interactions by resonant quartz crystal. Anal Chem. 2007;79(23):9039-9044.

2. Dultsev FN, Ostanin VP, Klenerman D. "Hearing" bond breakage. measurement of bond rupture forces using a quartz crystal microbalance. Langmuir. 2000;16(11):5036-5040.

3. Cooper MA, Dultsev FN, Minson T, Ostanin VP, Abell C, Klenerman D. Direct and sensitive detection of a human virus by rupture event scanning. Nat Biotechnol. 2001;19:833-837.

4. Edvardsson M, Rodahl M, Kasemo B, Höök F. A dual-frequency QCM-D setup operating at elevated oscillation amplitudes. Anal Chem. 2005;77(15):4918-4926.

5. Billy MD. On the low vibration modes observed in a sphere submitted to a tangential contact force. Ultrasonics. 2006;45(1-4):127-132.

6. Chatterjee A, Cusumano JP, Zolock JD. On contact-induced standing waves in rotating tires: experiment and theory. J Sound Vib. 1999;227(5): 1049-1081.
7. Murthy Peri MD, Cetinkaya C. Non-contact microsphere-surface adhesion measurement via acoustic base excitations. J Colloid Interface Sci. 2005;288(2):432-443.

8. Brukman MJ, Carpick RW. Vibrations of the "beetle" scanning probe microscope: identification of a new mode, generalized analysis, and characterization methodology. Rev Sci Instrum. 2006;77(3): 033706-033712.

9. Matsagar VA, Jangid RS. Influence of isolator characteristics on the response of base-isolated structures. Eng Struct. 2004;26(12): $1735-1749$.

10. Sauerbrey G. The use of quartz oscillators for weighing thin layers and for microweighing. Z Phys. 1959;155:206-222.

11. van der Werff MJ, Yuan YJ, Hirst ER, Xu WL, Chen H, Bronlund JE. Quartz crystal microbalance induced bond rupture sensing for medical diagnostics. IEEE Sensors J. 2007;7(5):62-769.

12. Raymer MG. The silicon web: physics for the Internet age. New York: Taylor \& Francis Group; 2009.

13. Almasriv AM. Characterization of double walled carbon nanotubes polyvinylidene fluoride nanocomposites. Dissertation, Office of Graduate Studies of Texas A\&M University. 2006:10-11.

14. Yuan YJ, Zhao Y, Xu WL. Characterization of molecular interactions of an immobilized biotinylated monolayer and streptavidin-coated microspheres by bond-rupture scanning. Anal Chim Acta. 2010; 680(1-2):59-64.

15. Edvardsson M, Rodahl M, Höök F. Investigation of binding event perturbations caused by elevated QCM-D oscillation amplitude. Analyst. 2006;131(7):822-828

16. Schmid A, Fujii S, Armes SP. Polystyrene-silica nanocomposite particles via alcoholic dispersion polymerization using a cationic azo initiator. Langmuir. 2006;22(11):4923-4927.

17. Borovsky B, Mason BL, Krim J. Scanning tunneling microscope measurements of the amplitude of vibration of a quartz crystal oscillator. J Appl Phys. 2000;88(7):4017-4021.

18. Grubmuller H, Heymann B, Tavan P. Ligand binding: molecular mechanics calculation of the streptavidin-biotin rupture force. Science. 1996;271(5251):997-999.

19. Freitag S, Trong IL, Chilkoti A, Klumb LA, Stayton PS, Stenkamp RE. Structural studies of binding site tryptophan mutants in the high-affinity streptavidin-biotin complex. J Mol Biol. 1998;279:211-221. 


\section{Appendices}

Appendix I: A piezoelectric model of thickness shear mode

Most AT-cut TSM piezoelectric quartz crystals are excited with a vertical field as follows:

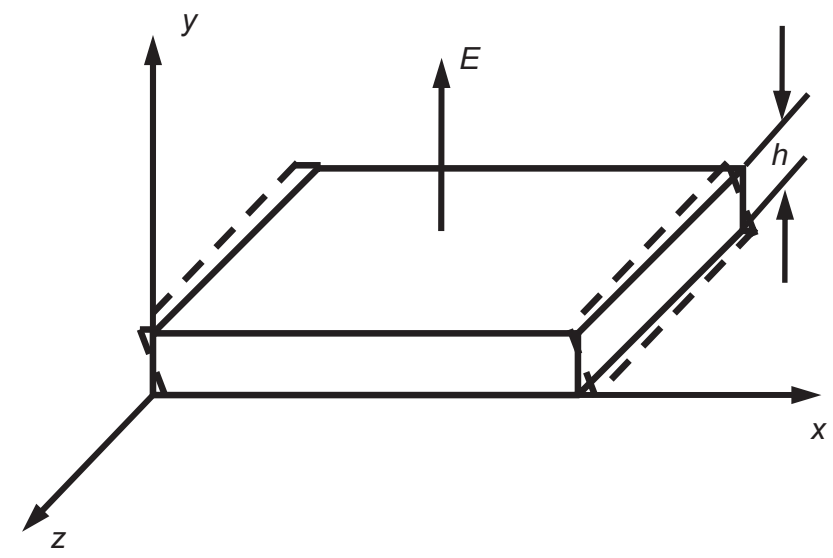

Under electric field $E$ along the $y$-direction, thickness shear vibration generated on the $z$ surface, where piezoelectric constant is $e_{26}$, and elastic stiffness constant is $c_{66}^{D}$. The density of the quartz is $\rho$. The displacement of the quartz along the $x$-axis, $u$, can be described by the one-dimensional wave equation:

$$
\frac{\partial^{2} u}{\partial t^{2}}=c^{2} \frac{\partial^{2} u}{\partial y^{2}}
$$

Here $c$ is the wave velocity $\left(c=\sqrt{c_{66}^{D} / \rho}\right)$. The general solution for the wave equation is:

$$
u=(A \cos k y+B \sin k y) \exp (j \omega t)
$$

Here $k=\omega / c$.

The fourth class piezoelectric equation of the AT-cut TSM quartz is defined as follows: ${ }^{1}$

$$
\begin{gathered}
E_{2}=-h_{26} S_{6}+D_{2} / \mathcal{E}_{22} \\
T_{6}=c_{66}^{D} S_{6}-h_{26} D_{2}
\end{gathered}
$$

Here $E_{2}$ is the electric field along the y-direction, and $T_{6}$ is the shear stress in the $x y$ plane. $h_{26}$ is the piezoelectric constant matrix, and $\varepsilon_{22}$ is the dielectric constant matrix. Shear strain in the $x y$ plane is $S_{6}=\partial u / \partial y$, and $D_{2}$ is the electric displacement along the y-direction. If the electric field $E_{2}$ is given as:

$$
E_{2}=E=E_{0} \exp (j \omega t)
$$

Then, according to Eq. 3, the electric displacement $D_{2}$ is:

$$
\begin{aligned}
D_{2} & =\varepsilon_{22}\left(E_{2}+h_{26} S_{6}\right) \\
& =\varepsilon_{22}\left(E_{0}+h_{26}(-A k \sin k y+B k \cos k y)\right) \exp (j \omega t) \\
& =D_{0} \exp (j \omega t)
\end{aligned}
$$

According to Eq. 4, the shear stress $T_{6}$ is:

$T_{6}=\left(c_{66}^{D}(-A k \sin k y+B k \cos k y)-h_{26} D_{0}\right) \exp (j \omega t)$

At the free surface, the boundary condition is:

$$
T_{6 \cdot y=0}=T_{6 \cdot y=l}=0
$$

Substitute Eq. 6 into Eq. 7, then obtain A and B. Substituting A and B into Eq. 2, the solution for AT-cut TSM crystal wave equation is:

$$
u=\frac{h_{26} D_{0}}{c_{66}^{D} k}\left[\frac{\cos k(h-y)-\cos k y}{\sin k h}\right] \exp (j \omega t)
$$

The $x$-axial displacement $u$ of the chip surface, ie, when $y=h$, is:

$$
u=A \exp (j \omega t)
$$

Here $A=h_{26} D_{0} / c_{66}^{D} k[1-\cos k h / \sin k h]$, is the maximum displacement of a TSM chip.

\section{Appendix 2: A mechanical model of the interaction of microspheres and QCM surface}

If a streptavidin-coated microsphere is bound on the QCM surface vis biotin-streptavidin interaction, ${ }^{2}$ a uniform microsphere is approximately attached to the QCM surface through a flexible bond. The sphere's vibration can be considered a mechanical mode of the sphere rotating around the vertex as shown:

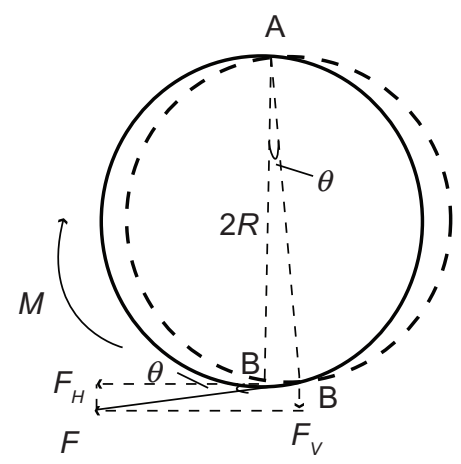


The surface displacement of QCM is at a few nanometers or several tens of nanometers, as reported by Edvardsson et al. ${ }^{3}$ With regard to the microsphere radius at the micron level, it can be assumed that the bottom of the microsphere vibrates with the quartz crystal. In order to take pivot vibration into account, it is necessary to postulate the fixed top point of a microsphere. The microsphere vibrates around the vertex $\mathrm{A}$. The displacement of the bottom $\mathrm{B}$ is determined by the horizontal displacement of the piezoelectric plate's surface, shown in Eq. 9:

$$
u=A \exp (j \omega t)
$$

Then the line speed of point B is given as follows:

$$
v(t)=\frac{d u(t)}{d t}=j A \omega \exp (j \omega t)
$$

The angular acceleration of point $B$ is:

$$
\beta(t)=\frac{1}{R} \frac{d v(t)}{d t}=-\frac{A}{R} \omega^{2} \exp (j \omega t)
$$

The rotational moment around point $\mathrm{A}$ is:

$$
M(t)=I \beta(t)=-I \frac{A}{R} \omega^{2} \exp (j \omega t)
$$

Here $I$ is the moment of inertia rotating around point A. The model of calculation of the moment of inertia is illustrated as follows:

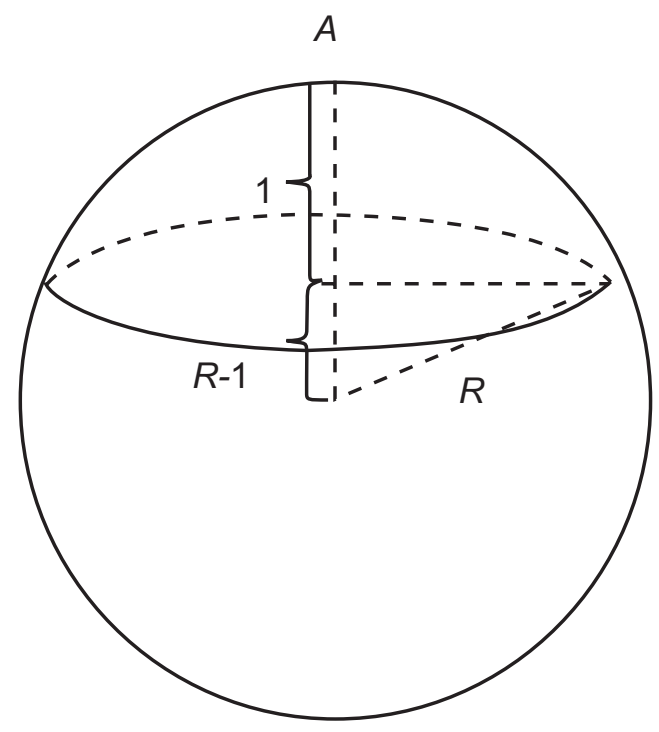

Therefore, the moment of inertia rotating around vertex A is given as follows:

$$
\begin{aligned}
I= & \int_{0}^{2 R} l^{2} d m \\
= & \pi \rho \int_{0}^{2 R} l^{2}\left[R^{2}-|R-l|^{2}\right] d l \\
= & \pi \rho \int_{0}^{2 R}\left(2 R l^{3}-l^{4}\right) d l \\
= & \pi \rho \frac{1}{2} R l^{4}-l^{5}{ }^{2 R} \\
& -\pi \rho R^{5} \\
& -m R^{2}
\end{aligned}
$$

Then the rotational torque is:

$$
M(t)=-I \frac{A}{R} \omega^{2} \exp (j \omega t)=-\frac{6}{5} m R A \omega^{2} \exp (j \omega t)
$$

Moment $M=F \cdot l=F \cdot 2 R$, so the force $F$ that the TSM chip drives the microsphere to vibrate is:

$$
F(t)=\frac{M(t)}{2 R}=-\frac{3}{5} m A \omega^{2} \exp (j \omega t)
$$

The angle between $F$ and horizontal direction is:

$$
\begin{gathered}
\tan \theta=\frac{u}{2 R}=\frac{A}{2 R} \exp (j \omega t) \\
\sin \theta=\frac{u}{\sqrt{u^{2}+4 R^{2}}}=\frac{A \exp (j \omega t)}{\sqrt{A^{2} \exp (j 2 \omega t)+4 R^{2}}}
\end{gathered}
$$

The vertical component of $F$ is:

$$
F_{v}(t)=F(t) \sin \theta=-\frac{3}{5} m A^{2} \omega^{2} \frac{\exp (j 2 \omega t)}{\sqrt{A^{2} \exp (j 2 \omega t)+4 R^{2}}}
$$

When $\exp (j 2 \omega t)=1$, the vertical component $F_{v}$ reaches the maximum. That is, $t=n \pi / \omega, \mathrm{n}=0,1,2,3 \ldots \ldots$,

$$
\left|F_{v \max }\right|=\frac{3}{5 \sqrt{A^{2}+4 R^{2}}} m A^{2} \omega^{2}
$$

As is shown in Figure 3, bond-rupture events happened between the microspheres and QCM. The forces exerted on the molecular bond include $F^{\prime}$ by the microsphere, the horizontal force $F_{q h}$, and the vertical force $F_{q v}$ by QCM's surface, and gravity $G_{2}$ (negligible). The maximum 
vertical force $F_{B R}$ exerted on the molecular bond by the microsphere is:

$$
\left|F_{B R \max }\right|=\left|-F_{v \max }\right|=\frac{3}{5 \sqrt{A^{2}+4 R^{2}}} m A^{2} \omega^{2}
$$

If the streptavidin-coated microsphere was a uniform polystyrene microsphere, its mass is:

$$
m=\frac{4}{3} \pi \rho R^{3}
$$

Here polystyrene's density $\rho$ is $1050 \mathrm{~kg} / \mathrm{m}^{3}{ }^{4}$

Borovsky et $\mathrm{al}^{5}$ measured the relationship between the amplitude of the QCM and the voltage by experiments:

$$
A=C Q V_{d}
$$

where $C=1.4 \times 10^{-12}$ is a constant determined experimentally, $Q$ the QCM merit factor ( $Q$ factor), and $V_{d}$ is the driving voltage.

By substituting Eq. 21 and 22 into Eq. 20, we can find the relationship between bond rupture force and particle radius, the applied voltage and frequency at the QCM electrode center.

$F_{B R}=\frac{4 \pi \rho R^{3}\left(C Q V_{d}\right)^{2} \omega^{2}}{5 \sqrt{\left(C Q V_{d}\right)^{2}+4 R^{2}}}=\frac{16 \pi^{3} \rho R^{3}\left(C Q V_{d}\right)^{2} f_{0}^{2}}{5 \sqrt{\left(C Q V_{d}\right)^{2}+4 R^{2}}}$

\section{Appendix 3: The relationship between bond rupture force $F_{B R}$ and the particle radius $R$}

If QCM resonance frequency $\left(f_{0}\right)$ was $10 \mathrm{MHz}$; $Q$ factor $(Q)$ 2500; drive voltage $\left(V_{d}\right) 10 \mathrm{~V}$, the amplitude $(A)$ would be $35 \mathrm{~nm}$ according to Eq. 22. Under this scenario, the bond rupture force $F_{B R}$ is:

$$
F_{B R}=\frac{1.274 \times 10^{4} R^{3}}{\sqrt{1.225 \times 10^{-15}+4 R^{2}}}
$$

The relationship between the bond-rupture force and particle radius is shown in Appendix 1.

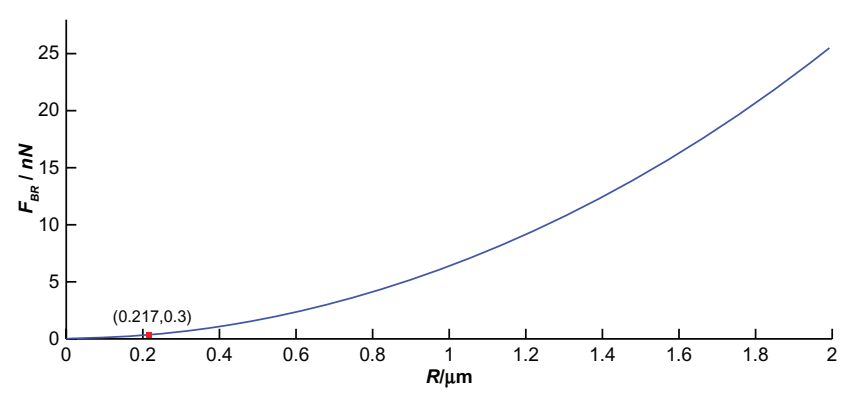

Appendix 4: The relationship between bond-rupture force $F_{B R}$ and QCM resonant frequency $f_{0}$

If the microsphere radius ( $R$ ), QCM $Q$ factor $(Q)$, and driving voltage $\left(V_{d}\right)$ were chosen as $1 \mu \mathrm{m}, 2500 \mathrm{~V}$, and $10 \mathrm{~V}$, respectively; according to Eq. 23, the bond rupture force $F_{B R}$ is:

$$
F_{B R}=6.370 \times 10^{-23} f_{0}^{2}
$$

The relationship between the bond-rupture force and the resonant frequency is shown in Appendix 2.

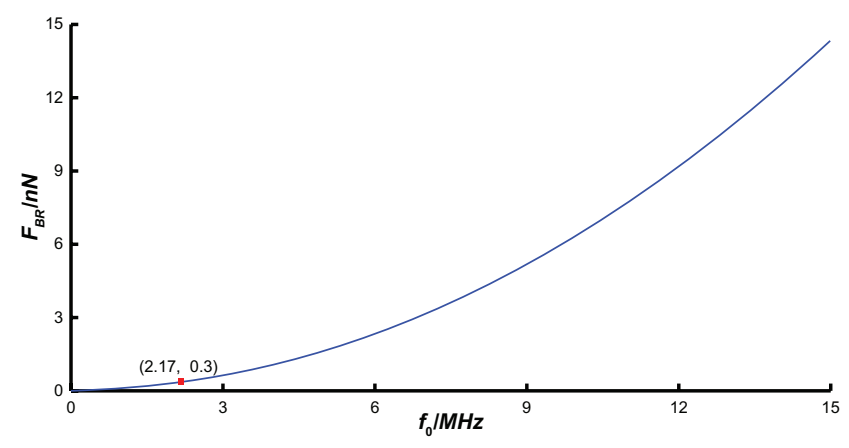

\section{Appendix 5:The relationship between bond-} rupture force $F_{B R}$ and driving voltage $V_{d}$

If the microsphere radius $(R), \mathrm{QCM} Q$ factor $(Q)$, and resonant frequency $\left(f_{0}\right)$ were chosen as $1 \mu \mathrm{m}, 2500 \mathrm{MHz}$, and $10 \mathrm{MHz}$, respectively; then the bond rupture force $F_{B R}$ is:

$$
F_{B R}=\frac{1.274 \times 10^{-10} V_{d}^{2}}{\sqrt{1.225 \times 10^{-5} V_{d}^{2}+4}}
$$

The relationship between the bond-rupture force and the driving voltage is shown in Appendix 3.

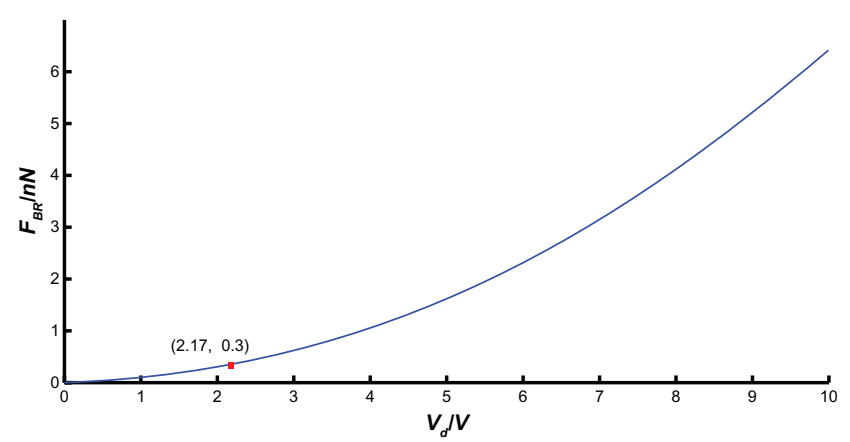




\section{References}

1. Almasriv AM. Characterization of double walled carbon nanotubes polyvinylidene fluoride nanocomposites [dissertation]. College Station, TX: Office of Graduate Studies of Texas A\&M University; 2006:10-11.

2. Yuan YJ, Zhao Y, Xu WL. Characterization of molecular interactions of an immobilized biotinylated monolayer and streptavidin-coated microspheres by bond-rupture scanning. Anal Chim Acta. 2010;680(1-2): 59-64.

3. Edvardsson M, Rodahl M, Höök F. Investigation of binding event perturbations caused by elevated QCM-D oscillation amplitude. Analyst. 2006;131(7):822-828.
4. Schmid A, Fujii S, Armes SP. Polystyrene-silica nanocomposite particles via alcoholic dispersion polymerization using a cationic azo initiator Langmuir. 2006;22(11):4923-4927.

5. Borovsky B, Mason BL, Krim J. Scanning tunneling microscope measurements of the amplitude of vibration of a quartz crystal oscillator. J Appl Phys. 2000;88:4017-4021.

International Journal of Nanomedicine

\section{Publish your work in this journal}

The International Journal of Nanomedicine is an international, peerreviewed journal focusing on the application of nanotechnology in diagnostics, therapeutics, and drug delivery systems throughout the biomedical field. This journal is indexed on PubMed Central, MedLine, CAS, SciSearch $®$, Current Contents ${ } /$ Clinical Medicine,
Journal Citation Reports/Science Edition, EMBase, Scopus and the Elsevier Bibliographic databases. The manuscript management system is completely online and includes a very quick and fair peer-review system, which is all easy to use. Visit http://www.dovepress.com/ testimonials.php to read real quotes from published authors.

Submit your manuscript here: http://www.dovepress.com/international-journal-of-nanomedicine-journal 